\title{
SHAREv2: fluctuations and a comprehensive treatment of decay feed-down $\dagger$
}

\author{
G. Torrieri ${ }^{a}$, S. Jeon ${ }^{a, b}$, J. Letessier ${ }^{c, d}$, and J. Rafelski ${ }^{d}$ \\ a Department of Physics, McGill University, Montreal, QC H3A-2T8, Canada \\ ${ }^{b}$ RIKEN-BNL Research Center, Upton NY, 11973, USA \\ ${ }^{c}$ Laboratoire de Physique Théorique et Hautes Energies ${ }^{\ddagger}$ \\ Université Paris 7, 2 place Jussieu, F-75251 Cedex 05, France \\ ${ }^{d}$ Department of Physics, University of Arizona, Tucson, AZ 85721, USA
}

\begin{abstract}
This the user's manual for SHARE version 2. SHARE [1] (Statistical Hadronization with Resonances) is a collection of programs designed for the statistical analysis of particle production in relativistic heavy-ion collisions. While the structure of the program remains similar to v1.x, v2 provides several new features such as evaluation of statistical fluctuations of particle yields, and a greater versatility, in particular regarding decay feed-down and input/output structure. This article describes all the new features, with emphasis on statistical fluctuations.
\end{abstract}

Keywords: Heavy ion collisions, Statistical models, Fluctuations

PACS: 24.10.Pa, 24.60.-k, 25.75.Dw, 24.60.Ky, 25.75.Nq, 12.38.Mh

Updates available from: http://www.physics.arizona.edu/〜 torrieri/SHARE/share.html and the authors upon request.

$\dagger$ Work supported by U.S. Department of Energy grant DE-FG02-04ER41318, the Natural Sciences and Engineering research council of Canada, the Fonds Nature et Technologies of Quebec. G.T. thanks the Tomlinson foundation for support. S.J. thanks RIKEN BNL Center and U.S. Department of Energy [DEAC02-98CH10886] for providing facilities essential for the completion of this work. The authors wish to express their gratitude to Lucy Carruthers for invaluable assistance in debugging and adapting the software described in this work.

$\ddagger$ LPTHE, Univ. Paris 6 et 7 is: Unité mixte de Recherche du CNRS, UMR7589. 


\section{Contents}

1 Introduction $\quad 5$

1.1 Evaluation of yields and fluctuations . . . . . . . . . . . . . . . . . 7

1.2 Chemical potential and chemical non-equilibrium . . . . . . . . . . . . . 8

1.3 Resonance decays . . . . . . . . . . . . . . . . . . . . . . . . 9 9

1.4 Volume fluctuations and fluctuations of ratios . . . . . . . . . . . . . . . 9

1.5 Fluctuations and detector acceptance . . . . . . . . . . . . . . . . . . 10

1.6 Correlations, resonances and detector acceptance . . . . . . . . . . . . . 11

2 Implementation of GCE fluctuations in SHAREv2 12

3 Decay feed-down and particle yields 12

3.1 Particle decay acceptance data files . . . . . . . . . . . . . . . . . . . . 12

3.2 Compatibility with SHAREv1.x experimental data files . . . . . . . . . . . . . 15

4 Quark chemistry $r$

4.1 Charm mesons . . . . . . . . . . . . . . . . . . . . . . . . . . 16

5 User Interface files and new commands 16

5.1 New single file control . . . . . . . . . . . . . . . . . . . . 16

5.2 Combining data points . . . . . . . . . . . . . . . . . . . . . 17

5.3 Miscellaneous . . . . . . . . . . . . . . . . . . . . . 18

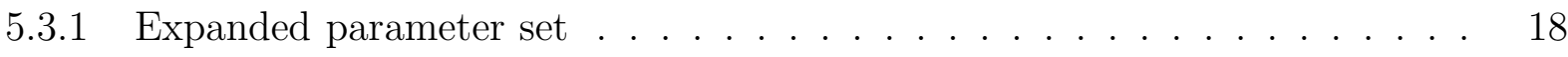

5.3.2 Data point sensitivity analysis . . . . . . . . . . . . . . . 18

5.3.3 Data point sensitivity profiles . . . . . . . . . . . . . . . . . 19

5.3.4 Additional output in $\chi^{2}$ and statistical significance profiles . . . . . . . . 19

5.3.5 Improved treatment of fit errors . . . . . . . . . . . . . . . . 19

6 Comparison with previous versions 20

6.1 Testing SHAREv2 . . . . . . . . . . . . . . . . . . . . . 20

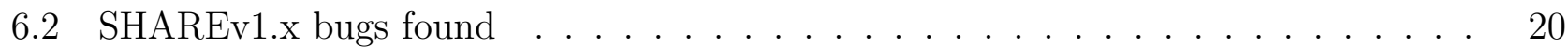

$\begin{array}{llr}7 & \text { Installation } & 21\end{array}$

8 Status, conclusions, and future plans $r$ 


\section{Program Summary}

Title of the program: SHAREv2,

April 2006

Computer:

PC, Pentium III, 512MB RAM

not hardware dependent;

Operating system:

Linux: RedHat 6.1, 7.2, FEDORA etc.

not system dependent;

Programming language: FORTRAN77

Size of the package: $167 \mathrm{~KB}$ directory, without libraries (see

http://wwwasdoc.web.cern.ch/wwwasdoc/minuit/minmain.html

http://wwwasd.web.cern.ch/wwwasd/cernlib.html

for details on library requirements ).

Distribution format: tar gzip file

Number of lines in distributed program (.tar file), including test data, etc.: 26017

Keywords: fluctuations, relativistic heavy-ion collisions, particle production, statistical models, decays of resonances

Computer: Any computer with an $\mathrm{f} 77$ compiler

Nature of the physical problem:

Event-by-event fluctuations have been recognized to be the physical observable capable to constrain particle production models. Therefore, consideration of event-by-event fluctuations is required for a decisive falsification or constraining of (variants of) particle production models based on (grand-, micro-) canonical statistical mechanics phase space, the so called statistical hadronization models (SHM).

As in the case of particle yields, to properly compare model calculations to data it is necessary to consistently take into account resonance decays. However, event-by-event fluctuations are more sensitive than particle yields to experimental acceptance issues, and a range of techniques needs to be implemented to extract 'physical' fluctuations from an experimental event-by-event measurement.

Method of solving the problem:

The techniques used within the SHARE suite of programs [1] are updated and extended to fluctuations. A full particle data-table, decay tree, and set of experimental feed-down coefficients are provided. Unlike SHAREv1.x, experimental acceptance feed-down coefficients can be entered for any resonance decay.

SHAREv2 can calculate yields, fluctuations, and bulk properties of the fireball from provided thermal parameters; alternatively, parameters can be obtained from fits to experimental data, via 
the MINUIT fitting algorithm [2]. Fits can also be analyzed for significance, parameter and data point sensitivity.

Averages and fluctuations at freeze-out of both the stable particles and the hadronic resonances are set according to a statistical prescription, calculated via a series of Bessel functions, using CERN library programs. We also have the option of including finite particle widths of the resonances. A $\chi^{2}$ minimization algorithm, also from the CERN library programs, is used to perform and analyze the fit. Please see [1] for more details on these.

\section{Purpose:}

The vast amount of high quality soft hadron production data, from experiments running at the SPS, RHIC, in past at the AGS, and in the near future at the LHC, offers the opportunity for statistical particle production model falsification. This task has turned out to be difficult when considering solely particle yields addressed in the context of SHAREv1.x [1]. For this reason physical conditions at freeze-out remain contested. Inclusion in the analysis of event-by-event fluctuations appears to resolve this issue. Similarly, a thorough analysis including both fluctuations and average multiplicities gives a way to explore the presence and strength of interactions following hadronization (when hadrons form), ending with thermal freeze-out (when all interactions cease).

SHAREv2 with fluctuations will also help determine which statistical ensemble (if any), e.g., canonical or grand-canonical, is more physically appropriate for analyzing a given system. Together with resonances, fluctuations can also be used for a direct estimate of the extent the system reinteracts between chemical and thermal freeze-out.

We hope and expect that SHAREv2 will contribute to decide if any of the statistical hadronization model variants has a genuine physical connection to hadron particle production.

Computation time survey:

We encounter, in the FORTRAN version computation, times up to seconds for evaluation of particle yields. These rise by up to a factor of 300 in the process of minimization and a further factor of a few when $\chi^{2} / \mathrm{N}_{\text {DoF }}$ profiles and contours with chemical non-equilibrium are requested.

\section{Accessibility:}

The program is available from:

- The CPC program library,

- The following website:

http://www.physics.arizona.edu/ ${ }^{2}$ torrieri/SHARE/share.html

- from the authors upon request.

\section{SUMMARY OF NEW FEATURES (w.r.t. SHAREv1.x)}

Fluctuations: In addition to particle yields, ratios and bulk quantities SHAREv2 can calculate, fit and analyze statistical fluctuations of particles and particle ratios;

Decays: SHAREv2 has the flexibility to account for any experimental method of allowing for decay feed-downs to the particle yields; 
Charm flavor: Charmed particles have been added to the decay tree, allowing as an option study of statistical hadronization of $J / \psi, \chi_{c}, D_{c}$, etc.;

Quark chemistry: Chemical non-equilibrium yields for both $u$ and $d$ flavors, as opposed to generically light quarks $q$, are considered; $\eta-\eta^{\prime}$ mixing, etc., are properly dealt with, and chemical non-equilibrium can be studied for each flavor separately;

Misc: Many new commands and features have been introduced and added to the basic user interface. For example, it is possible to study combinations of particles and their ratios. It is also possible to combine all the input files into one file.

\section{SHARE Compatibility and Manual:}

This write-up is an update and extension of [1]. The user should consult Ref. [1] regarding the principles of user interface and for all particle yield related physics and program instructions, other than the parameter additions and minor changes described here. SHAREv2 is downward compatible for the changes of the user interface, offering the user of SHAREv1 a computer generated revised input files compatible with SHAREv2. 


\section{Introduction}

The statistical hadronization model [3-6] (SHM) assumes particles are created according to their phase space weight, given the locally available energy and quantum numbers. Such a reaction model implies that the underlying dynamics of strong interactions saturates the strength of each particle production quantum matrix element.

This approach can be used to calculate the event-by-event average, as well as fluctuation (distribution width) and higher cumulants of any 'soft' observable. Event-by-event particle fluctuations have been subject to intense current theoretical [7-15], and experimental interest [16-19]. SHAREv2 will offer a standardized framework to evaluate these.

While qualitative study of fluctuations is useful as a test of new physics, a quantitative analysis including both average multiplicities and their event-by-event fluctuations constitutes a powerful probe of hadronization conditions [20-23]. In particular, the following questions can be addressed when both yields and fluctuations are considered in the same model framework:

- SHM can be falsified if and when fluctuations do not scale w.r.t. averages as expected in statistical physics. Moreover, only if the same set of thermal parameters gives good description of experimentally measured yields and fluctuations, can we claim that the SHM fit is physically sound.

- As has recently been shown [24-28], the value to which the scaled variance $\sigma_{N}$ (see Eq. (2)) for a single particle converges in the thermodynamic limit varies by as much as an order of magnitude when different statistical ensembles are considered. Thus, fluctuations can help decide if and when certain particle yields should be studied in grand canonical or canonical ensembles.

- SHM fits containing both the average particle multiplicity and the fluctuation de-correlate the hadronization temperature $T$ and light quark phase space occupancy $\gamma_{q}$ (see Eq. 11 and [1]) typical of fits when only the average multiplicities are fitted [20].

Therefore, the study of both fluctuations and yields can help to experimentally distinguish between the chemical equilibrium freeze-out model $\left(T \simeq 170 \mathrm{MeV}, \gamma_{q}=1\right.$ [29]), or the best fit with chemical non-equilibrium at typically lower $T$ [30].

- Considering the directly detectable resonance decays, fluctuations of particle yield ratios offer a way to quantitatively gauge the effect of hadronic re-interactions between formation and thermal freeze-out [21].

To investigate these questions, it is necessary, in evaluation of both particle yields and fluctuations to:

- Incorporate all particles resonance decay trees [31] in the program structure;

- Obtain particle yields and fluctuations for a given set of thermodynamic parameters; 
- i) Check if the parameters obtained by fitting particle yields are consistent with observed fluctuations;

ii) Once all corrections to fluctuations due to experimental setup are understood, incorporate the fluctuations along with yields into the chemical freeze-out fitting procedure.

SHAREv2 comprises a framework that addresses these challenges.

As implied above, event-by-event particle yield fluctuations are subject to many subtle experimental effects which need to be understood and kept under control for a joint yield-fluctuation analysis to proceed. Further, there is the choice of statistical model ensemble in computation of the phase space volume:

1) Evaluation with exact energy and discrete quantum number conservation (micro-canonical ensemble - MCE);

2) In the canonical ensemble (CE), statistical energy fluctuations are allowed, conserving discrete quantum number(s) exactly;

3) In the grand-canonical ensemble (GCE), statistical fluctuations of all conserved quantities occur - there are also mixed CE-GCE ensembles where some particle yields are conserved and other fluctuate.

Clearly, the fluctuations of particle yields are most constrained in MCE and least constrained in GCE. Thus, although in the three ensembles, the first moments of any observable distribution, i.e., expectation values, coincide in the thermodynamic limit, this will not be the case for the fluctuations [24-26]. The choice of appropriate ensemble in the situation considered has to be made based on evaluation of prevailing physical conditions.

In study of total particle yields, in the physical context of heavy ion collisions, the electrical charge and baryon number are fixed and, in these variables, we have to consider the $\mathrm{CE}$ or MCE if and when we are observing all particles. On the other hand, if we only observe a sub-volume of the system, which is exchanging energy and particles with an unobserved 'bath' consisting of the remainder of the reaction system, then, also conserved quantum numbers must be allowed to fluctuate, which implies use of the GCE for all observables. However, when the totality of the produced particles carrying a conserved quantum number falls within the detector acceptance region, occasional detection of one such particle implies presence of the corresponding anti-particle, and thus in that case $\mathrm{CE}$ or MCE must be applied.

Within the context of heavy ion physics, with reactions occurring at large energy, a study of fluctuations within a narrow momentum rapidity ${ }^{1}$ acceptance window provides for the division between 'system' and 'bath', with the bath being the unobserved rapidity domain. In all experiments currently capable to measure fluctuations, detector acceptance is limited typically to the central rapidity phase space coverage. Such an acceptance domain in the boost invariant (denoted below as subscript b.i.) limit is equivalent to a configuration space sub-volume [32] and thus for both particle ratios, and particle yield width (fluctuation) [23], we have:

$$
\frac{\left\langle N_{i}\right\rangle_{\mathrm{GC}}}{\left\langle N_{j}\right\rangle_{\mathrm{GC}}}=\frac{\left(d N_{i} / d y\right)_{\mathrm{b} . \mathrm{i} .}}{\left(d N_{j} / d y\right)_{\mathrm{b} . \mathrm{i} .}}
$$

\footnotetext{
${ }^{1}$ The rapidity $y$, defined by $y=\frac{1}{2} \ln \left(\frac{E+p_{z}}{E-p_{z}}\right)$, where $E$ is the particle energy and $p_{z}$ the particle momentum component parallel to the collision axis, is a variable additive under Lorentz transformations parallel to this axis.
} 
Here, $\left\langle N_{i}\right\rangle$ is the event-by-event average of particle $i, d N_{i} / d y$ is the average number of particles in an element of rapidity at central rapidity.

Similarly, barring acceptance effects (discussed in sections 1.5 and 1.6 of this work, as well as in [23]), the scaled variance, defined as

$$
\sigma_{X}^{2}=\frac{\left\langle(\Delta X)^{2}\right\rangle}{\langle X\rangle}=\frac{\left\langle X^{2}\right\rangle-\langle X\rangle^{2}}{\langle X\rangle},
$$

will be given by

$$
\sigma_{N_{i}}^{2}=\left(\frac{d \sigma_{N_{i}}^{2}}{d y}\right)_{\text {b.i. }} .
$$

We conclude that, in experiments with limited central rapidity acceptance, both yields and fluctuations should be evaluated in the GCE with respect to the conserved quantum numbers (charge $Q$, baryon number $b$, strangeness $s-\bar{s}$ ).

Use of the GCE, for at least some conserved charges, is required by the experimental observation of a significant fluctuation in those charges [16-18]. This fluctuation has been found to be compatible with Poisson scaling,

$$
\left\langle(\Delta N)^{2}\right\rangle \sim\langle N\rangle
$$

which is approximately followed by the GCE fluctuations. This is not the only scaling known to be present in this area of physics. Elementary reaction systems have been observed to follow a non-Poissonian scaling $[33,34]$ w.r.t. multiplicity averages,

$$
\left\langle(\Delta N)^{2}\right\rangle \sim\langle N\rangle+c\langle N\rangle^{2}
$$

where $c$ is a constant. As has been argued previously, [35-37], it is possible to describe this scaling by considering an extension of the Grand Canonical ensemble (variously referred to as Isobaric or Pressure ensemble) where system volume is also allowed to fluctuate.

In SHAREv2, we consider only GCE yields and fluctuations and search to explore whether the grand canonical statistical hadronization model can quantitatively reproduce fluctuations in the same way as it was shown to reproduce particle yields in heavy ion A-A reactions.

\subsection{Evaluation of yields and fluctuations}

In GCE, particle yields and fluctuations can be calculated by a textbook method [38]. For a hadron with an energy $E_{p}=\sqrt{p^{2}+m_{i}^{2}}$, the energy state occupancy is,

$$
n_{i}\left(E_{p}\right)=\frac{1}{\Upsilon_{i}^{-1} e^{E_{p} / T} \pm 1},
$$

where the upper sign is for fermions and the lower sign is for bosons. The chemical fugacity $\Upsilon_{i}$ will be considered in section 1.2. 
The yield average is obtained by multiplying the occupancy number Eq. 6 by the density of states (where $V$ is volume and $g$ degeneracy):

$$
\left\langle N_{i}\right\rangle=g V \int \frac{d^{3} p}{(2 \pi)^{3}} n_{i}\left(E_{p}\right)
$$

The fluctuation in this number is found to be:

$$
\left\langle\left(\Delta N_{i}\right)^{2}\right\rangle=\left.\Upsilon_{i} \frac{\partial\left\langle N_{i}\right\rangle}{\partial \Upsilon_{i}}\right|_{T, V}=g V \int \frac{d^{3} p}{(2 \pi)^{3}} n_{i}\left(E_{p}\right)\left(1 \mp n_{i}\left(E_{p}\right)\right) .
$$

Eqs. (7-8) can be evaluated to any desired accuracy by converting them into an expansion of Bessel function terms [5],

$$
\begin{aligned}
\left\langle N_{i}\right\rangle & =\frac{g V T^{3}}{2 \pi^{2}} \sum_{n=1}^{\infty} \frac{( \pm 1)^{n-1} \Upsilon_{i}^{n}}{n^{3}} W\left(\frac{n m_{i}}{T}\right), \quad \Upsilon_{i} e^{-m_{i} / T}<1 \\
\left\langle\left(\Delta N_{i}\right)^{2}\right\rangle & =\frac{g V T^{3}}{2 \pi^{2}} \sum_{n=1}^{\infty} \frac{( \pm 1)^{n-1} \Upsilon_{i}^{n}}{n^{3}}\left(\begin{array}{c}
2+n-1 \\
n
\end{array}\right) W\left(\frac{n m_{i}}{T}\right)
\end{aligned}
$$

where $W(x)=x^{2} K_{2}(x)$ (see [1], section 2, for the technical details required in doing these calculations, as well as a discussion of particles with finite width).

\subsection{Chemical potential and chemical non-equilibrium}

The particle fugacity $\Upsilon_{i}$ can be obtained from the quark number content of the particle as well as the fugacities and phase space occupancies of the individual quark flavors. If a particle has $N_{q}=N_{u}^{i}+N_{d}^{i}, N_{s}^{i}$ and $N_{c}^{i}$, light up + down, strange and charm quarks, $N_{\bar{q}}^{i}=N_{\bar{u}}^{i}+N_{\frac{i}{d}}^{i}, N_{\bar{s}}^{i}$ and $N_{\bar{c}}^{i}$ antiquarks, and isospin $I_{3}$, the fugacity $\Upsilon_{i}$, or, equivalently the associated particle chemical potential $\mu_{i}$ will be given by:

$$
\Upsilon_{i}=e^{\mu_{i} / T}=\left(\lambda_{u} \gamma_{u}\right)^{N_{u}^{i}}\left(\lambda_{d} \gamma_{d}\right)^{N_{d}^{i}}\left(\lambda_{s} \gamma_{s}\right)^{N_{s}^{i}}\left(\lambda_{c} \gamma_{c}\right)^{N_{c}^{i}}\left(\lambda_{\bar{u}} \gamma_{\bar{u}}\right)^{N_{\bar{u}}^{i}}\left(\lambda_{\bar{d}} \gamma_{\bar{d}}\right)^{N_{\bar{d}}^{i}}\left(\lambda_{\bar{s}} \gamma_{\bar{s}}\right)^{N_{\bar{s}}^{i}}\left(\lambda_{\bar{c}} \gamma_{\bar{c}}\right)^{N_{\bar{c}}^{i}}
$$

where $\lambda_{\bar{i}}=\lambda_{i}^{-1}$, and $\gamma_{\bar{i}}=\gamma_{i}$. The individual $u, d$ light quark variables are related to the SHARE $q$ and $I_{3}$ variables $\lambda_{q}=\sqrt{\lambda_{u} \lambda_{d}}$ and $\lambda_{I_{3}}=\lambda_{u} / \lambda_{d}$, see Ref. [1], and similarly $\gamma_{q}=\sqrt{\gamma_{u} \gamma_{d}}$ and $\gamma_{3}=$ $\gamma_{u} / \gamma_{d}$, see Eq.(23) below. The condition of chemical equilibrium, for a flavor $f$, imposes $\gamma_{f}=1$ [38]. The assumption of chemical equilibrium is not automatic in a dynamically expanding system with a possible phase transition and, in fact, good theoretical arguments have been proposed for $\gamma_{q, s} \neq 1$ for a range of energies (see [30], and references therein). However, it is difficult, using fits to particle yields, to distinguish between two models based on different temperatures and $\gamma$ values.

For instance, models based on both a higher freeze-out temperature and $\gamma_{q}=1$ [29] or a lower freeze-out temperature and $\gamma_{q}>1$ [30] have been used to fit SPS and RHIC data. As shown in [23], this ambiguity is resolved when both yields and fluctuations can be considered. 
A complication arises for hadrons such as the $\pi^{0}$ or the $\eta$, which are in a flavor superposition state. If $\gamma_{u, d, s} \neq 1$, the yield of the hadron with fractional flavor content is considerably altered by the mixing. For a meson of fractional quark number structure,

$$
\left|i>=\alpha_{u}\right| u \bar{u}>+\alpha_{d}\left|d \bar{d}>+\alpha_{s}\right| s \bar{s}>, \quad \alpha_{u}^{2}+\alpha_{d}^{2}+\alpha_{s}^{2}=1,
$$

the fugacity comprises the chemical yield fugacities as follows:

$$
\Upsilon_{i}=\lambda_{i}\left(\gamma_{u}^{2} \alpha_{u}^{2}+\gamma_{d}^{2} \alpha_{d}^{2}+\gamma_{s}^{2} \alpha_{s}^{2}\right) .
$$

Fractional flavor content has non-negligible influence on the abundances of $\eta^{0}$ and $\eta^{\prime}$, and their decay products, in fits which allow for chemical non-equilibrium factor $\gamma_{s}$. The same remarks applies when $\gamma_{3}=\gamma_{u} \gamma_{d}^{-1} \neq 1$ to $\pi^{0}, \rho^{0}$, etc. Thus, $\gamma_{3} \neq 1$ can considerably enhance $\pi^{0} \propto \gamma_{3}^{2}+\gamma_{3}^{-2}$ yield, while maintaining $\pi^{ \pm}$yields symmetry (since $\gamma_{3}$ cancels out in $\pi^{ \pm}$).

Importantly, the evolution of quark-coalesced hadrons into final quark-eigenstates hadrons (like the oscillation of neutral kaons into $\mathrm{K}_{S}$ and $\mathrm{K}_{L}$ ) means that the 'source' QGP quark content will not, in general, be equal to the 'final' hadron quark content.

\subsection{Resonance decays}

Eqs. 7 and 8 can be used to calculate the event-by-event averages and fluctuations of all hadrons at hadronization. This, however, is quite different from the observed averages and fluctuations, since most hadrons are strong resonances (unstable states), which decay after freeze-out, either to stable particles or to other resonances. The final state particle yields can be computed by taking the effect of these feed-downs into account [6].

The ensemble average of the total yield $\left\langle N_{i}\right\rangle$ is:

$$
\left\langle N_{i}\right\rangle_{\text {total }}=\left\langle N_{i}\right\rangle_{\text {direct }}+\sum_{j \neq i} B_{j \rightarrow i}\left\langle N_{j}\right\rangle .
$$

$B_{j \rightarrow i}$ is the probability (branching ratio) for the decay products of $j$ to include $i$.

The fluctuation after resonance feed-down is given by:

$$
\left\langle\left(\Delta N_{j \rightarrow i}\right)^{2}\right\rangle=B_{j \rightarrow i}\left(\mathcal{N}_{j \rightarrow i}-B_{j \rightarrow i}\right)\left\langle N_{j}\right\rangle+B_{j \rightarrow i}^{2}\left\langle\left(\Delta N_{j}\right)^{2}\right\rangle .
$$

The second term corresponds to the fluctuation in the yield of resonances. The first term, in the number of $j \rightarrow i$ decays given the branching ratio $b_{j \rightarrow i} . \mathcal{N}_{j \rightarrow i}$ is the number of particles type $i$ produced in the decay, so that $\sum_{i} B_{j \rightarrow i}=\mathcal{N}_{j \rightarrow i}$.

$\mathcal{N}_{j \rightarrow i}=1$ for nearly all decays of nearly all resonances; The most significant exception are decays to multiple $\pi^{0} \mathrm{~s}$, such as $\eta \rightarrow 3 \pi^{0}$.

\subsection{Volume fluctuations and fluctuations of ratios}

The expression 10 neglects volume fluctuations, coming from centrality cuts and dynamics of system expansion. These are accounted for by dividing the observed fluctuation into an extensive 
and an intensive part,

$$
\left\langle(\Delta X)^{2}\right\rangle \approx\left\langle(\Delta x)^{2}\right\rangle\langle V\rangle^{2}+\langle x\rangle^{2}\left\langle(\Delta V)^{2}\right\rangle
$$

$\langle x\rangle,\left\langle x^{2}\right\rangle$ can be calculated by the statistical methods described in this section.

It is difficult to describe the volume fluctuation coefficient $\left\langle(\Delta V)^{2}\right\rangle$ in a model-independent way. The most straight forward way to deal with this problem is to choose observables insensitive to $\left\langle(\Delta V)^{2}\right\rangle$.

Any observable where $\left.\langle x\rangle^{2} \ll\langle\Delta x)^{2}\right\rangle$ would be a good candidate. This is why the fluctuation in electromagnetic charge has long been considered to be a promising observable [10].

A more general approach is to consider the event-by-event fluctuation of particle ratios [9], where the volume fluctuation $\left\langle(\Delta V)^{2}\right\rangle$ is zero by construction. Fluctuation of particles ratios can be calculated from the denominator's and numerator's fluctuation once the full resonance decay tree is known [9]. Note that, unlike in the case of particle yields, resonance decays produce both fluctuations and correlations, since a resonance can decay both into a numerator and a denominator particle. If this is the case, a high resonance admixture can considerably reduce the fluctuation of a ratio w.r.t. Poisson expectation.

The formulas to be used are, for the event-by-event fluctuation of the ratio of two particles $N_{1} / N_{2}$ (for example $K^{-} / \pi^{+}[9]$ ):

$$
\sigma_{N_{1} / N_{2}}^{2}=\frac{\left\langle\left(\Delta N_{1}\right)^{2}\right\rangle}{\left\langle N_{1}\right\rangle^{2}}+\frac{\left\langle\left(\Delta N_{2}\right)^{2}\right\rangle}{\left\langle N_{2}\right\rangle^{2}}-2 \frac{\left\langle\Delta N_{1} \Delta N_{2}\right\rangle}{\left\langle N_{1}\right\rangle\left\langle N_{2}\right\rangle} .
$$

The last correlation term is given by the resonance decay into both particles

$$
\left\langle\Delta N_{1} \Delta N_{2}\right\rangle=\left\langle N_{1} N_{2}\right\rangle-\left\langle N_{1}\right\rangle\left\langle N_{2}\right\rangle \simeq \sum_{j} B_{j \rightarrow 1,2}\langle N\rangle_{j}
$$

$\sigma_{N}^{2}$ does not depend on the average system volume $(\langle V\rangle)$, since it cancels between the numerator and the denominator. $\sigma_{N 1 / N 2}^{2}$, however, does acquire a dependence on $\langle V\rangle$ since ratio fluctuations scale as $\langle N\rangle^{-1}$. Hence, an analysis incorporating fluctuations of particle ratios should also consistently account for particle yields, and the system normalization (thermodynamic parameter norm, [1] section 3.1) should be considered as a fit parameter.

The equations presented in this section can be used to compute fluctuations of particles yields and ratios from given SHM parameters (temperatures, chemical potentials, and phase space occupancies). However, it has long been known [39] that fluctuations are considerably less robust than yields against systematic effects resulting from limited experimental acceptance. These effects, therefore, have to be taken into account within the SHM. The next two subsections give two such issues addressed within SHAREv2.

\subsection{Fluctuations and detector acceptance}

One way to separate detector acceptance effects from physics is to eliminate the former via mixed event techniques. A 'static' fluctuation $\sigma_{\text {stat }}^{2}$ is measured in a sample of fake events, constructed by 
using tracks from different events [16]. Since tracks from different events have no correlations or quantum corrections, $\sigma_{\text {stat }}^{2}$ is determined solely by a trivial Poisson contribution as well as detector acceptance effects.

Within the statistical hadronization model:

$$
\left(\sigma_{N_{i}}^{2}\right)_{\text {stat }}=1
$$

For particle ratios in mixed events, the correlation term, $\left\langle\Delta N_{1} \Delta N_{2}\right\rangle$ in Eq.17, vanishes, while $\left\langle\left(\Delta N_{1,2}\right)^{2}\right\rangle$ follow Poisson scaling. Hence, Eq. 17 reduces to

$$
\left(\sigma_{N_{i} / N_{j}}^{2}\right)_{s t a t}=\frac{1}{\left\langle N_{i}\right\rangle}+\frac{1}{\left\langle N_{j}\right\rangle}
$$

The 'dynamical fluctuation' $\sigma_{d y n}^{2}[9,39-41]$ corresponds to the difference between the 'raw' total fluctuation $\sigma^{2}$ and the fake event fluctuation:

$$
\sigma_{d y n}^{2} \equiv \sqrt{\sigma^{2}-\sigma_{\text {stat }}^{2}}
$$

In certain limits, $\sigma_{d y n}^{2}$ can be shown [39] to be independent of detector acceptance. Hence, comparing SHM estimates of $\sigma_{d y n}$ to experimental measurements is more reliable than using $\sigma^{2}$.

\subsection{Correlations, resonances and detector acceptance}

Because mixed event tracks are uncorrelated, mixed event techniques cannot account for detector acceptance effects within particle correlations. Thus, the branching ratios appearing in Eqs. 14 and 15 need to be modified, $B_{j \rightarrow i} \rightarrow \alpha_{j \rightarrow i} B_{j \rightarrow i}$, and in addition Eq. 17 needs to be updated,

$$
\sigma_{N_{1} / N_{2}}^{2}=\frac{\left\langle\left(\Delta N_{1}\right)^{2}\right\rangle}{\left\langle N_{1}\right\rangle^{2}}+\frac{\left\langle\left(\Delta N_{2}\right)^{2}\right\rangle}{\left\langle N_{2}\right\rangle^{2}}-2 \alpha_{12} \frac{\left\langle\Delta N_{1} \Delta N_{2}\right\rangle}{\left\langle N_{1}\right\rangle\left\langle N_{2}\right\rangle}
$$

here $\alpha_{j \rightarrow i}$ refers to the probability that particle $i$ will end up in the detector's acceptance region given particle $j$ is in the region, while $\alpha_{12}$ measures the probability that both decay products will be inside this region.

For a boost invariant azimuthally complete system, $\alpha_{j \rightarrow i}=1$ since particles leaving the detector acceptance region will be balanced by particles entering. However, in general $\alpha_{12}<1$, since if a resonance is outside the detector acceptance region both particles can not be inside it, and the intrinsic particle decay momentum adds a rapidity scale to the system, breaking boost invariance $[20]$.

See [20] for an illustration of how to calculate $\alpha_{12}$. While such a comprehensive calculation is outside the scope of the current version of the program, we offer the user the possibility of entering an $\alpha_{12}$ for any resonance decay as an input parameter. See section 3 on how to do this. In practice, this should only be necessary for a few most frequent and energetic resonance decays, such as $\rho \rightarrow \pi \pi$ and $K^{*} \rightarrow K \pi$. 


\section{Implementation of GCE fluctuations in SHAREv2}

Experimental event-by-event fluctuation data points were implemented in the SHARE interface in a similar manner as yield and ratio data points (see [1], section 3.4). The tag which denotes that a fluctuation is being calculated is fluct_yld. A statement such as

particle1 fluct_yld data $\Delta_{\text {stat }} \Delta_{\text {syst }}$ fit? will calculate $\sigma_{N}^{2}$ of particle1 (defined in Eq. 2).

If fit? is set to 1 , this data point is used within a fit together with the experimentally measured value data and the statistical $\left(\Delta_{\text {stat }}\right)$ and systematic $\left(\Delta_{\text {syst }}\right)$ error . The format of the data-line is exactly the same as in SHAREv1.x ( [1] section 3.4).

To calculate the fluctuation of a ratio, particle1 should be substituted by the data point number where the ratio is defined. For instance, if the 5 th data point (from the top) is a $K^{-} / \pi^{-}$ ratio, than it's fluctuation is given by:

$05 \quad$ fluct_yld data $\Delta_{\text {stat }} \Delta_{\text {syst }}$ fit?

(See section 5.2 of this paper for a more general treatment of this data point referencing).

SHAREv2 implements most definitions of dynamical fluctuations used to date by experimental collaborations. These are implemented as additional tags of fluct_xxx type, where $\mathbf{x x x}$ refers to different ways the experimental measurement is presented.

The possible types of data points are:

fluct_dyn To calculate $\sigma_{d y n}=\sqrt{\sigma^{2}-\sigma_{\text {stat }}^{2}}$, as measured in [41],

fluct_dnr To calculate $\sigma_{d y n}=\frac{\sigma^{2}}{\sigma_{\text {stat }}^{2}}$ as suggested in [9].

\section{$3 \quad$ Decay feed-down and particle yields}

\subsection{Particle decay acceptance data files}

As shown in section 1, decay feed-down is a fundamental component of the statistical hadronization model. However, the limited coverage of most detectors means that the feed-down coefficients will acquire an experimental correction, corresponding to the probability that the decay products of a given resonance formed within the detector acceptance region will also be in that region. As shown in section 1.6, these corrections need to be considered when calculating both fluctuations and yields.

Weak decays, such as $\Lambda \rightarrow p \pi^{-}$(most protons at RHIC are, in fact, given by feed-down from hyperons), are particularly susceptible to experimental acceptance, as they occur at a macroscopic distance from the primary vertex. Hence, weak experimental feed-down corrections include a geometrical as well as a momentum space component.

Since the 'parent' particles are not always directly observed, SHARE must be able to compute final hadron multiplicities including experimental feed-down coefficients for all decays where this effect is non-negligible. 
SHAREv1.x allowed the user to input experimental (weak) feed-down contributions to produced particle yields via four acceptance coefficients:

$K_{S} \rightarrow$ anything, $K_{L} \rightarrow$ anything, $Y \rightarrow$ Mesons, $Y \rightarrow$ baryons (see [1], section 3.4.1).

It turns out this approach was not sufficiently flexible: for instance, undetected $\Sigma \rightarrow N \pi$ feed-down can be treated very differently from $\Lambda \rightarrow p \pi^{-}$corrections, considering the difference in lifespan, and (vertex) acceptance cuts applied.

Moreover, the experimental acceptance of different hyperon $\rightarrow$ nucleon weak decays, such as $\Xi^{-} \rightarrow \Lambda \pi^{-}$as compared to $\Lambda \rightarrow p \pi^{-}$is likely to be considerably different. Finally, different weak decays of the same hadron can have varying acceptances, compare $K_{L} \rightarrow 3 \pi$, with $K_{L} \rightarrow \pi e \nu$, and with $K_{L} \rightarrow \pi \mu \nu$. A similar acceptance problem may arise in special cases involving strong decay chains when the acceptance region is particularly narrow and/or the particles rapidity distribution is not well understood.

A more flexible way of treating weak decay contributions to particle yields is therefore necessary. Specifically, there should be an easy way to allow for any arbitrary decay/reaction contributing to any data point. SHAREv2 provides such a possibility through user defined decay feed-down files.

In data file containing the experimental results to be fitted (see [1] section 3.4.3), a weak decay control file is now signaled by a statement of the type:

\section{Weakdecay File.feed}

where File.feed is a 9-letter filename. The program then obtains the decay acceptance weights from File.feed, an ASCII file in a format similar to the decay tree files (described in [1] section 3).

Fig. 1, and the attached input files provided with the SHARE package, show how to implement the weak decay acceptance coefficients. While many weak feed-down files might be involved in the same analysis, generally, they are experiment-specific, and hence can be kept track of in a systematic way. Alternatively, all weak feed-down files and experimental data-files can be combined in a single large file, using the methods described in section 5.1 of this paper.

In more detail, a typical line in a feed-down file will be:

Parent Daughter Daughter $_{2}$ all/1st/2nd/cor coeff or, for 3-body decays,

Parent Daughter Daughter $_{2}$ Daughter $_{3}$ all/1st/2nd/3rd/cor coeff The switch all/1st/2nd/3rd refers to the daughter to which the decay coefficient applies.

all means that the decay coefficient is the same for all daughters, while $\mathbf{1 s t} / \mathbf{2 n d} / \mathbf{3 r d}$ means only the 1st/2nd/3rd daughter will be removed from the experimental yield. For example, in the $\Lambda \rightarrow \pi p$ decay in STAR $[42,43]$, STAR accepts the nucleon from the $\Lambda$ decay but not the $\pi$, and this fine tuning of the decay is clearly quite important as a relatively large fraction of all nucleons comes from weak $\Lambda$ decays.

cor refers to the fractional contribution of the acceptance to the two particle correlation $\left\langle\right.$ Daughter $_{1}$ Daughter $\left._{2}\right\rangle$ induced by a common resonance decay from parent, denoted as $\alpha_{12}$ in Eq. 22 (section 1.6).

SHARE will renormalize the decay Parent $\rightarrow$ all/1st/2nd/3rd/cor by the coefficient coeff when calculating all data points after the given weakdecay statement.

It is therefore possible to assign a different weak decay file to each data point, or assume that a 


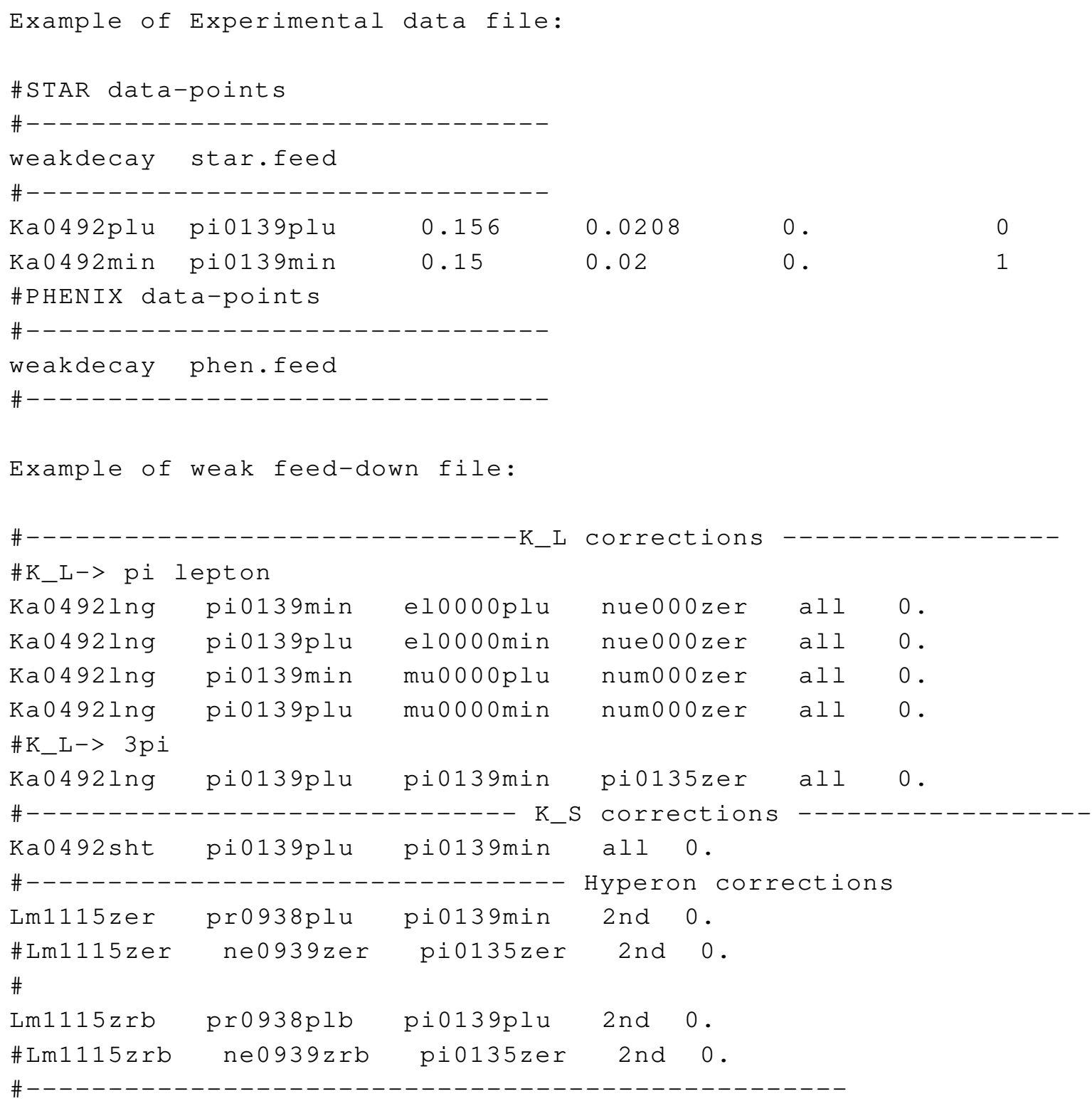

Figure 1: An example of the SHAREv2 weak feed-down acceptance coefficient implementation.

group of data points are subject to the same set of weak decay yield contribution, (e.g., in general all data points from the same experiment should have the same weak decay file). The way to do this is the same as in v1.x ( [1] section 3.4.3): when the program reads a weakdecay statement, it assigns the current decay pattern to each data point encountered until a new weakdecay statement is met.

Two special case exist, for which no File.feed file is needed: 
weakdecay UNCORRECT 'uncorrected' (from the perspective of experimental data set) means that all weak decays contributions to particle yields are fully accepted by SHAREv2.

weakdecay NOWK_FEED means that all particle yields are computed without contributions from weak decays, from the perspective of experimental data this means that either all weak decay products are not accepted and/or have been all corrected for in experimental yields, as, e.g., applies to some NA49 results.

When fluctuations are considered, it is important to deal carefully with experimental corrections, which are neither close to total (close $100 \%$ accepted) nor null (close to $0 \%$ accepted). Weak decay corrections of the daughter particles are usually correlated with each other in momentum space, so the straight-forward application of Eq. 8 will not be a good description of fluctuations with a non-trivial detector acceptance function [13]. In this case, it is better to use dynamical, rather than total, fluctuations as discussed in section 1.5.

\subsection{Compatibility with SHAREv1.x experimental data files}

The improved weak decay treatment does not impair compatibility of experimental input files between SHAREv2 and SHAREv1.x. SHAREv2 will read a SHAREv1.x experimental data-file, and automatically calculate applicable contributions for each weak decay based on the information contained in the SHAREv1.x weakdecay statement. A line will be printed within the sharerun.out output file that signals a SHAREv1.x format weakdecay statement was encountered.

In addition, an output v2 weakdecay file called weak\#v1.x (where \# refers to the data point number) is automatically generated translating the v1.x weak decay information into v2 format. The user is advised to eventually change all weakdecay lines to

weakdecay weak\#v1.x

as the v2 format is considerably more powerful and less amenable to systematic error stemming from an incomplete understanding of weak decays.

\section{Quark chemistry}

SHAREv1.x input files listed particle chemical content by total isospin $I$ and its third component, $I_{3}$, as well as the number of light ( $q$, either $u$ or $d$ ), strange $(s)$ and charm $(c)$ quarks (See section 3.2 of [1]). In SHAREv2, $u$ and $d$ quarks are now separately accounted for. The particle listing format is:

name mass width spin $I \quad I 3 \quad u \quad d \quad s \quad a u$ ad as $c$

where name is the particle's 9-character name, $I$ and $I_{3}$ are the total and third component of the isospin, $\mathbf{u}, \mathbf{d}, \mathbf{s}, \mathbf{c}$ are the numbers of up, down, strange and charm quark numbers, while au, ad, as, ac are the respective anti quark numbers. The format of the table is otherwise identical to that discussed in [1], section 3.2.

To check for the possibility that phase space occupancy differs for the up and down quarks, a statistical model fit parameter (see [1], section 3.1) gam3 $\left(\gamma_{3}\right)$ has been introduced, such that:

$$
\gamma_{u}=\gamma_{q} \gamma_{3}, \quad \gamma_{d}=\gamma_{q} / \gamma_{3}
$$


The quark/anti quark numbers can be fractional, to account for the superposition states described at the end of section 1.2.

To calculate $(u, d, s)$ quark abundance in the statistically hadronizing QGP system (different, in general, from the freeze-out content, as shown in section 1.2), new bulk variables tot_u_qgp, tot_d_qgp and tot_s_qgp were introduced. These can be used in the same way as other bulk variables (see [1], section 3.4.2).

\subsection{Charm mesons}

Charmed particles have now been added in the files particles.data and partnowdt.data. Their nomenclature follows the general structure as described in [1], section 3.2. Dcxxxxxxx refer to $D_{c}$ mesons, Dsxxxxxxx, chixxxxcc to $\chi_{c}$ states and psixxxxcc to $J / \psi$ states.

Their abundance is regulated by the chemical potential $\lambda_{c}$ and the phase space occupancy $\gamma_{c}$, described in [1], section 3.1.

\section{$5 \quad$ User Interface files and new commands}

\subsection{New single file control}

As described in detail in [1], section 3, SHARE relies on quite a few input files.

- The run-file sharerun.data

- The particles list

- The particles decay tree

- The initial values of the thermodynamic parameters

- The experimental data points

- Initialization for each fit parameter

This structure makes it easy to quickly explore regions of parameter space within an analysis in progress. However, this system makes it easy to mistakenly lose a successfully completed and saved analysis, since a change in each of the files could considerably alter the end result. The introduction of weak decay correction file (see section 3) aggravates this problem.

SHAREv2, therefore, makes it possible to combine some, or all, input files into a single file. Once the user found an optimum analysis, all input files involved in it can be combined into one large sharerun.data file, which can be easily kept for future reproduction and modification.

This is done by changing the extension (.data or .feed) of the filename into .HERE. If the program encounters a filename ending in .HERE, it assumes the relevant input is immediately following the given line within the currently read file. The subsequent format is assumed to be unchanged from what it would have been had a separate file been opened (comments, etc.). The 


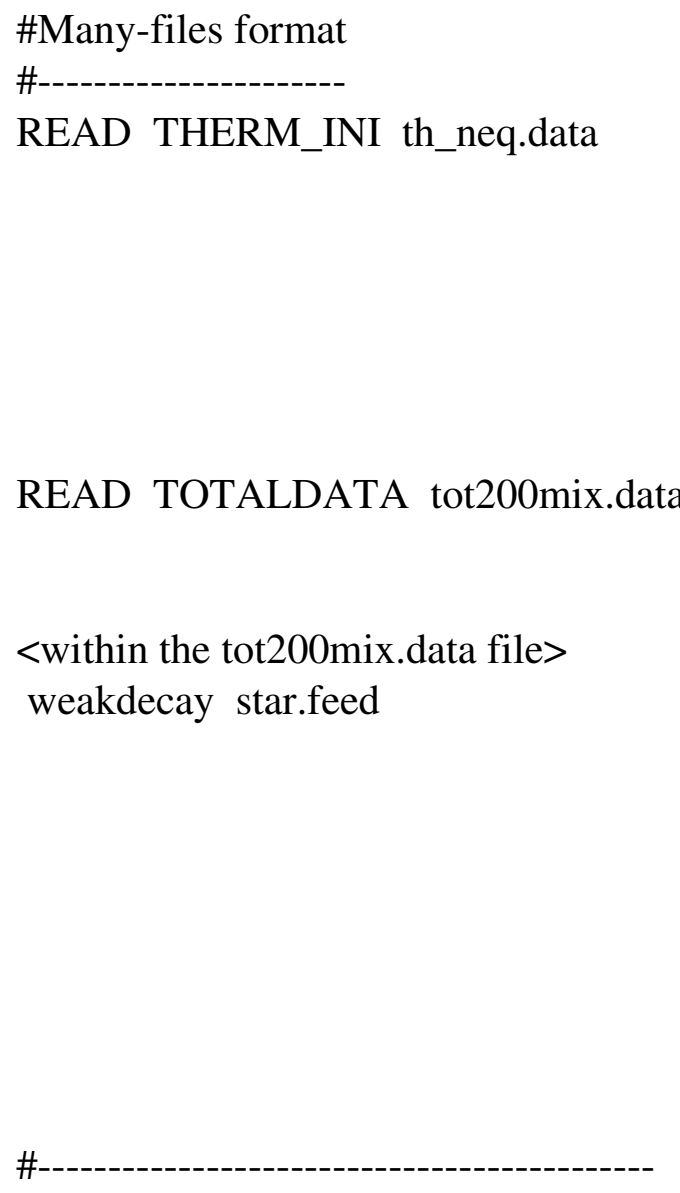

CALC FITRATIOS fitnw20M._neq
\#One-file format

\#-

READ THERM_INI th_neq.HERE

\#---- content of thermal file starts

temp 0.14

$\cdots$

accu $\quad 0.01$

*

\#--- content of thermal file ends

READ TOTALDATA tot200mix.HERE

\#--- content of experiment file starts

pi0139plu prt_yield $286.424 .2 \quad 0 . \quad 1$

...

weakdecay star.HERE

\#---- content of weak decay file starts

...

Ka492sht pi0139plu pi0139min all 0.7

$\cdots$

$*$

\#---- content of weak decay file ends

*

\#--- content of experimental file ends

\#--

CALC FITRATIOS fitnw20M._neq

Figure 2: Left: sharerun.data calling other input files. Right: One-file format.

only difference is that a $*$ symbol on a new line has to be present at the point where the separate file would have ended. When the program encounters the $*$ symbol, it switches back to reading the 'earlier' file, that is prior to the insert .HERE.

See Fig 2, and the provided file sharerun.data_onefile, for an example of how this works.

\subsection{Combining data points}

SHAREv2 gives the possibility to refer to a different data point within the given fit, and/or combine two data points, in order to fit the sum or a product of two particles. This feature was described, in the case of fluctuations of ratios, in section 2 of this paper.

The referring data point consists of one or two (for a combination) numbers, corresponding to the position, in the input file, of the point(s) being referred to. 
Two numbers united by an operation sign $(+,-, \mathrm{X}, /)$ will add, subtract, multiply and divide two data points. For instance, if the first data points from the top of the file (see [1] section 3.4, for a detailed explanation of the format) are:

$\begin{array}{llllll}\text { Lm1115zer } & \text { pi0139min } & \text { Data } & \Delta_{\text {stat }} & \Delta_{\text {syst }} & \text { Fit? } \\ \text { Lm1115zrb } & \text { pi0139plu } & \text { Data } & \Delta_{\text {stat }} & \Delta_{\text {syst }} & \text { Fit? }\end{array}$
then,

\section{X02 prt_yield Data $\Delta_{\text {stat }} \quad \Delta_{\text {syst }} \quad$ Fit?}

will fit $(\Lambda \bar{\Lambda}) /\left(\pi^{+} \pi^{-}\right)$, while

$01 \quad$ fluct_dyn Data $\Delta_{\text {stat }} \Delta_{\text {syst }}$ Fit?

will fit the dynamical $\Lambda / \pi^{-}$fluctuation, as described in section 2 .

To fit $(\Lambda+\bar{\Lambda}) /\left(\pi^{+}+\pi^{-}\right)$(but NOT the separate yields), the input file will read:

$\begin{array}{llllll}\text { Lm1115zer } & \text { prt_yield } & \text { Data } & \Delta_{\text {stat }} & \Delta_{\text {syst }} & \mathbf{0} \\ \text { Lm1115zrb } & \text { prt_yield } & \text { Data } & \Delta_{\text {stat }} & \Delta_{\text {syst }} & \mathbf{0} \\ \text { pi0139plu } & \text { prt_yield } & \text { Data } & \Delta_{\text {stat }} & \Delta_{\text {syst }} & \mathbf{0} \\ \text { pi0139min } & \text { prt_yield } & \text { Data } & \Delta_{\text {stat }} & \Delta_{\text {syst }} & \mathbf{0} \\ \text { 01+02 } & \mathbf{0 3 + 0 4} & \text { Data } & \Delta_{\text {stat }} & \Delta_{\text {syst }} & \mathbf{1}\end{array}$

NOTE: SHARE was written in FORTRAN77. Feature mentioned in this subsection use implicitly recursive code. SHAREv2 has been tested on several compilers and platforms, and found to work. However, compilers and operating systems vary — we would like to know if and when you experience problems.

\subsection{Miscellaneous}

The following (small) modifications were made in SHAREv2 compared to SHAREv1:

\subsubsection{Expanded parameter set}

The expanded parameter set includes as noted before, Eq. (23), gam3 which allows to incorporate a different $u, d$-flavor phase space occupancy. A further new variable dvol describes statistical pressure ensemble fluctuations in volume (Section 1, Eq. (16)). The provided input file sets and fixes dvol to zero and gam3 to unity, since experimental measurements sensitive to these parameters have not as yet been published.

All details about how to configure these parameters, and fix or relax them in the context of fits to experimental data, are unchanged w.r.t. v1.x, described in [1] sections 3.1, 3.6 and 3.7

\subsubsection{Data point sensitivity analysis}

Command DFIT, within the file sharerun.data can turn on and off the given data point as a point to be fitted.

The syntax for this command is

DFIT [Datapoint n.] [Fit $(0 / 1)]$

where Datapoint n. refers to the data point's position in the experimental data file from the 
top, while $\boldsymbol{F i t}(\mathbf{0 / 1})$ turns this point on (1) or off (0) as a point to be fitted. For instance, the following input in sharerun.data:

READ TOTALDATA tot200mix.data

\section{DFIT 51}

\section{CALC FITRATIOS fitnw20M-kpi}

DFIT 50

\section{CALC FITRATIOS fitnw20M-nkpi}

performs two fits.

The first, saved in file fitnw20M-kpi uses the 5th data point in tot200mix.data when calculating the $\chi^{2}$ (to be minimized).

The second one, saved in file fitnw20M-nkpi, does not.

\subsubsection{Data point sensitivity profiles}

Command SNSPROFIL calculates the data point sensitivity. The sensitivity is defined as the ratio between the data point's SHM prediction for a given statistical parameter, and SHM prediction at the best fit value for that parameter.

The syntax of SNSPROFIL is the same as DATPROFIL in [1], section 4. The two commands operate in the same way: all parameters, except the one on the abscissa, are minimized at each point in the profile.

Thus, the command

\section{CALC SNSPROFIL temp 0.10 .21005}

will calculate a sensitivity profile for the temperature, going from 0.1 to $0.2 \mathrm{GeV}$, with 100 points, of the fifth data point within the experimental data-file.

\subsubsection{Additional output in $\chi^{2}$ and statistical significance profiles}

$\chi^{2}$ profile commands now output the following files:

name.log A fit output for each point in the $\chi^{2}$ profile, in the same format as the usual fit output file ( [1], Fig. 4).

name.chi2, name.stsg Commands SNSPROFIL and DATPROFIL also output the $\chi^{2}$ profile (extension *.chi2) and $P_{\text {true }}$ profile (extension *.stsg).

\subsubsection{Improved treatment of fit errors}

SHAREv2 automatically runs the MINOS algorithm [2] if the fit can not get a robust estimate of the errors. This results in a considerable improvement of error-handling. This update entails no changes in the user interface or output format. 


\section{Comparison with previous versions}

\subsection{Testing SHAREv2}

SHAREv2 was extensively tested for programming and physics errors:

- SHM Calculations and fit results for SPS and RHIC energies were verified to be equal between SHAREv2 and SHAREv1.x reference results;

- SHAREv2 reads SHAREv1.x weak decay input. The equivalence between the two treatments, when weak decay files are designed to reproduce SHAREv1.x format, was shown to all decimal places;

- Fluctuations of conserved quantities (such as $\left\langle(\Delta Q)^{2}\right\rangle$ ) were compared before and after resonance decays. The conservation of this quantity implies that the enhanced fluctuations after all resonances decayed are exactly balanced by multiplicity correlations between the resonance decay products. This holds true to two decimal places (up to two-step correlations arising from decays such as $K(1600) \rightarrow K(892) \pi \rightarrow K \pi \pi$. These correlations are not tracked by SHARE, but their contribution is below $1 \%$ ).

\subsection{SHAREv1.x bugs found}

While developing and testing SHAREv2, several minor bugs and choice issues were found in the previous version SHAREv1.x, The most noteworthy issues which lead to sometimes noticeable (beyond line width) changes in the results are:

SHAREv1.1, v1.2 The Bessel function series was incorrectly truncated for large $\gamma_{q}$ (close to pion B-E condensation);

SHAREv1.3 Quark flavor mixing error in calculation of mesons such as $\eta$ and $\phi$, for $\gamma_{q} \neq \gamma_{s}$;

SHAREv1.1-v1.3 The most relevant issue is actually not an error but lack of versatility in the handling of $\Sigma \rightarrow p \pi$ decays: $\Sigma$-particles decay weakly, like the $\Lambda$ s and the $\Xi$ s. However, unlike $\Lambda$ and $\Xi, \Sigma$-decays are not experimentally reconstructible since at least one of $\Sigma$-decay products is neutral. In general, SHAREv1.x particles from these decays were included in the yield count. However, it turned out that while some experiments had much less than full acceptance for these decays, other experiments, e.g., NA49, have removed $\Sigma \rightarrow p$ feeddown via Monte-Carlo simulations accounting for the experimental acceptance of the decay products, with $\Sigma$ yields obtained from the observed $\Lambda$-yields.

Working with patched SHAREv1.x, we realized that $\Sigma$-decay issue mattered in that some fits

got better allowing for a modified $\Sigma$ weak decay pattern. Issues, such as this one, prompted us to introduce a more general treatment of weak decay particle yield contributions in SHAREv2. 


\section{$7 \quad$ Installation}

The $^{2}$ SHAREv.2.1 program code and input files are contained in a tar.gz archive. The file sharev2.1.tar.gz is available at

http://www.physics . arizona.edu/ torrieri/SHARE/share.html

To unpack it, create a SHARE directory, put the archive in it, and execute the following commands:

gunzip sharev2.1.tar

tar -xf sharev2.1.tar

The following files will then be created, enough for a complete 'representative' run of SHARE:

decays.data The complete Particle Data Group decay tree (section 3.3 in [1]);

dec_no.data An empty decays file, useful for testing the program calculations (abundancies reduce to modified Bessel functions) as well as studying the role of resonances in stable particle ratios;

fortrat A shell script compiling (in f77) the FORTRAN code which should be modified depending on location of FORTRAN (g77 or f77 or f95) and CERN library of programs;

particles.data Particle properties, with full widths (section 3.2, in [1]));

partnowdt.data Particle properties, with no widths. Calculations with this input file require considerably less computational time, and it suffices when there are no resonances in the fit;

ratioset.data The FORTRAN fit input file (section 3.5, in [1]);

sharerun.data A 'representative' run input file (section 4, in [1]); including an analysis of fluctuations and yields similar to what was presented by members of our collaboration in [21];

sharerun.data_onefile The same file in the single file format, as explained in section 5.1;

samplefit200 A directory containing the output files generated by running the provided 'sharerun.data', as a debugging/comparison standard;

sharev2.1.f SHAREv1.1 FORTRAN code. The header contains information about bug fixes;

thermo.data A representative thermal parameter input file (section 3.1, in [1]). It is set to reasonable non-equilibrium fit values;

totbar200.data A representative data input file (section 3.4, in [1]) containing ratios, yields and fluctuations drawn from RHIC experiments, as of July 2004 (see references in [21]);

star.feed An example of a decay feed-down coefficients file. See section 3.

\footnotetext{
${ }^{2}$ This chapter is nearly identical to the READMEv2.html file found on the SHARE webpage
} 
Note that SHARE requires CERN libraries, to be downloaded separately from http://wwwasd.web.cern.ch/wwwasd/cernlib.html

The compiler statement (in file 'fortrat') is

fry -L/usr/local/cern/pro/lib - o sharev2.1.exe sharev2.1.f -lmathlib-lkernlib-lpacklib-C which assumes that the CERN libraries are in directory

/usr/local/cern/pro/lib

If this is not true on your system, fortrat should be changed accordingly.

Once the directory is unpacked, the program should be compiled with

./fortrat

After this, typing

./sharev2.1.exe

should produce a correct run with a detailed output which shows the program's capability. A copy

of these files, produced on our computer system, is also included in sharev2.1.tar.gz within a directory called samplefit200 for comparison with the files produced by the installed program. Several output files are produced, with the following names as default. The contents of each file are explained in detail in [1] section 4.

fit*.out Fit output files;

graph* Fit output graphics (experiment, fitted values, calculated values);

prof* $\chi^{2}$ profiles and correlation functions for the various fits.

See [1] about more details about these files's contents.

\section{Status, conclusions, and future plans}

One of the areas of current intense interest in the field of high energy heavy ion reactions is the understanding of the mechanisms of soft hadron production (chemical freeze-out), that is the study of how the energy confined in the central fireball turns into matter in a multi particle production process.

The SHARE suite of programs is an analysis tool of particle yields addressing the following questions:

- What is the chemical freeze-out temperature, chemical potentials and volume?

- What are the physical properties of the fireball which hadronizes?

- Is the hadron system in chemical equilibrium at freeze-out?

- What is the degree of re-interaction between hadronization and freeze-out?

The need for SHARE arises from recognition that the book keeping task involved in the correct application of the statistical hadronization model is considerable, often transcending the resources 
available to individual researchers. The current SHAREv2 program follows on SHAREv1.x [1] adding three significant novel features: a) flexible handling of particle decay feed-down, b) fluctuations, and c) complete $u, d, s, c$ flavor content treatment. We note that, since SHAREv1.x was released, another analysis package appeared, THERMUS [44]. THERMUS has more convenient setup for the experimental data analysis environment. On the other hand, THERMUS is not addressing many of the features SHARE offers, including chemical non-equilibrium and now in the current SHAREv2, fluctuations, and light flavor details.

SHARE allows an analysis of experimental data that can address indirectly questions related to the dynamics of the fireball evolution, since chemical non-equilibrium implies a fast hadronization. However, the analysis of particle yields cannot give results of greater precision than is inherent in the data it treats, and this is not yet good enough to resolve the question about the nature of SHM as seen in the recent references [29,30,45-47], and the possible relation to the onset of a phase transition, [48]. SHAREv2 offers the additional analysis feature, the fluctuation in hadron yields and ratios, which will help to settle these issues, should precise particle yield experimental data not become available. Moreover, SHAREv2 through the study of the consistency of particle yield and fluctuation, can test the SHM in depth.

The development of phenomenological tools capable of falsifying statistical hadronization models is, of course, far from over. Possible extensions of chemical freeze-out model, in future version of SHARE, might include a canonical ensemble module, allowing to test SHM chemical nonequilibrium in small physical systems, and the introduction of an opacity parameter to correct the yields of observed resonances [49]. Another possible future development would entail extending SHARE towards a detailed description of momentum distributions. This would be somewhat different from THERMINATOR [50] model which relies on scaling in rapidity, and thus, only applies to ultra high energy collisions. We note that particle momentum distributions are dependent, in addition to the physics incorporated in SHAREv2 also on the dynamical evolution of the emitting source, and on the degree of resonance rescattering following on the chemical freeze-out. Strategies how these two interwoven effects can be disentangled are being developed. For further discussion of these and related questions we refer to a recent review [51].

\section{References}

[1] G. Torrieri, S. Steinke, W. Broniowski, W. Florkowski, J. Letessier and J. Rafelski, Comput. Phys. Commun. 167, 229 (2005).

[2] F. James and M. Roos, Comput. Phys. Commun. 10, 343 (1975).

[3] E. Fermi, Prog. Theor. Phys. 5, 570 (1950).

[4] I. Pomeranchuk, Proc. USSR Academy of Sciences (in Russian) 43, 889 (1951).

[5] L. D. Landau, Izv. Akad. Nauk Ser. Fiz. 17 (1953) 51.

[6] R. Hagedorn, Suppl. Nuovo Cimento 2, 147 (1965).

[7] S. Jeon and V. Koch, "Event-by-event fluctuations," arXiv:hep-ph/0304012, In: Hwa, R.C. (ed.) et al.: Quark gluon plasma, Singapore 2004, pp 430-490.

[8] S. Jeon, V. Koch, K. Redlich and X. N. Wang, Nucl. Phys. A 697, 546 (2002).

[9] S. Jeon and V. Koch, Phys. Rev. Lett. 83, 5435 (1999).

[10] S. Jeon and V. Koch, Phys. Rev. Lett. 85, 2076 (2000). 
[11] M. Asakawa, U. W. Heinz and B. Muller, Phys. Rev. Lett. 85, 2072 (2000).

[12] S. Mrowczynski, Phys. Rev. C 57, 1518 (1998).

[13] C. Pruneau, S. Gavin and S. Voloshin, Phys. Rev. C 66, 044904 (2002).

[14] J. Zaranek, Phys. Rev. C 66, 024905 (2002).

[15] Q. H. Zhang, V. Topor Pop, S. Jeon and C. Gale, Phys. Rev. C 66, 014909 (2002).

[16] J. G. Reid [STAR Collaboration], Nucl. Phys. A 698611 (2002).

[17] J. Adams et al. [STAR Collaboration], Phys. Rev. C 68, 044905 (2003).

[18] K. Adcox et al. [PHENIX Collaboration], Phys. Rev. Lett. 89, 082301 (2002).

[19] C. Alt et al. [NA49 Collaboration], Phys. Rev. C 70, 064903 (2004).

[20] G. Torrieri, S. Jeon and J. Rafelski, arXiv:nucl-th/0510024.

[21] G. Torrieri, S. Jeon and J. Rafelski, arXiv:nucl-th/0509077.

[22] G. Torrieri, S. Jeon and J. Rafelski, arXiv:nucl-th/0509067.

[23] G. Torrieri, S. Jeon and J. Rafelski, Accepted for Publication, Physical Review C arXiv:nucl-th/0503026.

[24] V. V. Begun, M. I. Gorenstein, A. P. Kostyuk and O. S. Zozulya, Phys. Rev. C 71, 054904 (2005).

[25] V. V. Begun, M. I. Gorenstein and O. S. Zozulya, Phys. Rev. C 72, 014902 (2005).

[26] V. V. Begun, M. Gazdzicki, M. I. Gorenstein and O. S. Zozulya, Phys. Rev. C 70, 034901 (2004) [arXiv:nucl-th/0404056].

[27] F. Becattini, A. Keranen, L. Ferroni and T. Gabbriellini, Phys. Rev. C 72, 064904 (2005) [arXiv:nuclth/0507039].

[28] J. Cleymans, M. Stankiewicz, P. Steinberg and S. Wheaton, "The origin of the difference between multiplicities in e+ e- annihilation and heavy ion collisions," arXiv:nucl-th/0506027.

[29] F. Becattini, J. Manninen and M. Gazdzicki, arXiv:hep-ph/0511092.

[30] J. Letessier and J. Rafelski, arXiv:nucl-th/0504028.

[31] K. Hagiwara et al., Particle Data Group Collaboration, Phys. Rev. D 66, 010001 (2002), see also earlier versions, note that the MC identification scheme for most hadrons was last presented in 1996.

[32] J. Cleymans, K. Redlich, Phys. Rev. C 60, 054908 (1999).

[33] H. Heiselberg, Phys. Rept. 351, 161 (2001).

[34] Z. Koba, H. B. Nielsen and P. Olesen, Nucl. Phys. B 40, 317 (1972).

[35] M. I. Gorenstein, Yad. Fiz. 31 (1980) 1630.

[36] S. Mrowczynski, Z. Phys. C 27, 131 (1985).

[37] R. Hagedorn, Z. Phys. C 17, 265 (1983).

[38] See any advanced undergraduate or graduate-level book on statistical mechanics, e.g., K. Huang, John Wiley and Sons

[39] C. Pruneau, S. Gavin and S. Voloshin, Phys. Rev. C 66, 044904 (2002).

[40] M. Gazdzicki and S. Mrowczynski, Z. Phys. C 54, 127 (1992).

[41] S. Das [STAR Collaboration], "Event by event fluctuation in K/pi ratio at RHIC," arXiv:nuclex/0503023.

[42] O. Barannikova [STAR Collaboration], "Probing collision dynamics at RHIC," arXiv:nucl-ex /0403014.

[43] C. Adler et al. [STAR Collaboration], Phys. Rev. Lett. 89, 092301 (2002).

[44] S. Wheaton and J. Cleymans, arXiv:hep-ph/0407174.

[45] J. Rafelski, J. Letessier and G. Torrieri, "Centrality dependence of bulk fireball properties at RHIC," Phys. Rev. C 72, 024905 (2005) nucl-th/0412072

[46] J. Letessier and J. Rafelski, arXiv:nucl-th/0506044.

[47] J. Cleymans, H. Oeschler, K. Redlich and S. Wheaton, arXiv:hep-ph/0511094. 
[48] M. Gazdzicki, arXiv:nucl-ex/0512034.

[49] J. Rafelski, J. Letessier and G. Torrieri, Phys. Rev. C 64, 054907 (2001) [Erratum-ibid. C 65, 069902 (2002)] [arXiv:nucl-th/0104042].

[50] A. Kisiel, T. Taluc, W. Broniowski and W. Florkowski, arXiv:nucl-th/0504047.

[51] W. Florkowski, arXiv:nucl-th/0509039, Invited talk at QM2005, Budapest. 